\title{
Prevalence, awareness and control of hypertension in Malaysia from 1980-2018: a systematic review and meta-analysis
}

\begin{abstract}
Background: Hypertension is a common public health problem worldwide and is a well-known risk factor for increased risk of cardiovascular diseases, contributing to high morbidity and mortality. However, there has been no systematic review and meta-analysis of a multiethnic population such as that of Malaysia. Aim: To determine the trend in prevalence, awareness and control rate of hypertension in Malaysia. Methods: Systematic searches were conducted in six databases (PubMed, Scopus, Ovid, CINAHL, Malaysian Medical Repository and Malaysia Citation Index) for articles published between 1980 and 2018. Two authors reviewed the studies and performed quality assessment and data extraction independently. Pooled estimates of hypertension prevalence, awareness and control rate were calculated using the DerSimonianLaird random-effects model. Subgroup and sensitivity analyses were performed. Results: We included 56 studies involving a total of 241796 participants. The overall pooled prevalence of hypertension aged $\geq 18$ years was $29.7 \%$. The prevalence of hypertension was the lowest in the 1980s $(16.2 \%, 95 \%$ confidence interval (CI): 13.4, 19.0), increasing up to $36.8 \%$ (95\%CI: 6.1 , 67.5 ) in the $1990 \mathrm{~s}$, then decreasing to $28.7 \%$ (95\%CI: $21.7,35.8$ ) in the 2000 s and $26.8 \%$ (95\%CI: 21.3, 32.3) in the 2010s. The prevalence of awareness was 51.4\% (95\%CI: 46.6, 56.3), while $33.3 \%$ (95\%CI: 28.4, 38.2) of those on treatment had achieved control of their blood pressure. Conclusion: In Malaysia, three in ten adults aged $\geq 18$ years have hypertension, while four in ten adults aged $\geq 30$ years have hypertension. Five out of ten people are aware of their hypertension status and only one-third of those under treatment achieved control of their hypertension. Concerted efforts by policymakers and healthcare professionals to improve awareness and control of hypertension should be of high priority.
\end{abstract}

Keyword: Prevalence; Awareness; Control; Hypertension; Blood pressure; Malaysia; Systematic review; Meta-analysis 